\title{
Dairy food, calcium and vitamin D intake in pregnancy, and wheeze and eczema in infants
}

\author{
Y. Miyake*, S. Sasaki\# ${ }^{\#}$ K. Tanaka* and Y. Hirota
}

ABSTRACT: The present prospective study examined the association between maternal consumption of dairy products, calcium, and vitamin D during pregnancy and the risk of wheeze and eczema in the infants aged 16-24 months.

Subjects were 763 Japanese mother-child pairs. Data on maternal intake during pregnancy were assessed with a diet history questionnaire. Symptoms of wheeze and eczema were based on criteria of the International Study of Asthma and Allergies in Childhood.

Higher maternal intake of total dairy products, milk, cheese and calcium during pregnancy was significantly related to a decreased risk of infantile wheeze, but not eczema (adjusted ORs (95\% $\mathrm{Cl})$ between extreme quartiles were $0.45(0.25-0.79), 0.50(0.28-0.87), 0.51(0.31-0.85)$, and 0.57 (0.32-0.99), respectively). When maternal vitamin $D$ consumption during pregnancy was categorised into two groups using a cut-off point at the 25th percentile, children whose mothers had consumed $\geqslant 4.309 \mu \mathrm{g} \cdot$ day $^{-1}$ had a significantly reduced risk of wheeze and eczema (adjusted ORs (95\% Cl) were 0.64 (0.43-0.97) and 0.63 (0.41-0.98), respectively).

Higher consumption of calcium and dairy foods other than yoghurt during pregnancy may reduce the risk of infantile wheeze. Higher vitamin D intake during pregnancy may be protective against childhood wheeze and eczema.

KEYWORDS: Calcium, dairy, eczema, pregnancy, vitamin D, wheeze

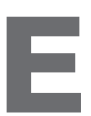
pidemiological studies of the relationship of intake of dairy products with asthma, wheeze, and/or eczema have provided mixed results [1-8]. Some studies found a protective relationship between dairy food intake and any symptoms of such conditions [1-3] whereas others reported a null association [4-7]. Only one cross-sectional study of young Australian adults showed a significant positive association between intake of dairy products other than milk and the prevalence of asthma [8].

The role of intra-uterine exposure in the development of allergic disorders has gained interest. A birth cohort study in Germany showed no associations between maternal intake of milk, yoghurt or cheese during pregnancy and the risk of doctordiagnosed eczema in the children at 2 yrs old [9]. In a birth cohort study in the Netherlands, maternal dairy intake (average of milk and milk product consumption) during pregnancy was not related to the risk of childhood wheeze during the first $8 \mathrm{yrs}$ of life [10]. A significant inverse exposure-response relationship between maternal vitamin $\mathrm{D}$ intake during pregnancy and the risk of recurrent wheeze but not eczema, was found in 3-yr-old children in the USA [11]. A prospective study in the UK found that higher maternal vitamin $\mathrm{D}$ consumption during pregnancy was significantly associated with a decreased risk of wheeze in children aged 5 yrs whereas such an inverse association was not observed in children at 2 yrs old [12]. Prenatal deficiency of vitamin D may affect fetal lung and immune system development [13]. Dairy foods are a rich source of calcium. To our knowledge, no epidemiological study has investigated the association between maternal calcium intake during pregnancy and the risk of childhood allergic disorders.

In this investigation, we examined the association between maternal consumption of dairy products, calcium, and vitamin D during pregnancy and the risk of wheeze and eczema in Japanese children at 16-24 months old, using data from the Osaka Maternal and Child Health Study (OMCHS).

\section{METHODS}

\section{Study population}

The OMCHS is a prospective cohort study. Details of the OMCHS have been described

\section{AFFILIATIONS}

*Dept of Public Health, Faculty of Medicine, Fukuoka University, Fukuoka,

\#Dept of Social and Preventive Epidemiology, School of Public Health, The University of Tokyo, Tokyo, and

"Dept of Public Health, Osaka City University Graduate School of Medicine, Osaka, Japan.

\section{CORRESPONDENCE}

Y. Miyake

Dept of Public Health

Faculty of Medicine

Fukuoka University

Fukuoka

Japan

E-mail: miyake-y@fukuoka-u.ac.jp

Received:

June 262009

Accepted after revision:

Sept 182009

First published online:

Oct 192009 
elsewhere [14]. We recruited pregnant females at baseline. Neyagawa City (Japan) is one of the 43 municipalities in Osaka Prefecture, a metropolis in Japan. Of the 3,639 eligible subjects in Neyagawa City, $627(17.2 \%)$ females participated in the OMCHS between November 2001 and March 2003. In order to increase the sample size, pregnant females living in municipalities other than Neyagawa City were also recruited. Eight pregnant females who lived in municipalities adjacent to Neyagawa City and who had become aware of the OMCHS at an obstetric clinic before August 2002 decided by themselves to participate in the OMCHS. Also, there were 77 participants who received explanations of the OMCHS from public health nurses in six other municipalities from August 2002 to March 2003. From October 2002 to March 2003, 290 participants were recruited from a university hospital and three obstetric hospitals in three other municipalities.

Finally, a total of 1,002 females between the 5th and 39th week of pregnancy gave their fully informed consent in writing and completed the baseline survey. Of the 1,002 females, 867 mother-child pairs participated in the second survey from 2 9 months post-partum. Of the 867 mother-child pairs, 763 participated in the third survey from 16-24 months postpartum and were included in the present analysis. The OMCHS was approved by the ethics committee of the Osaka City University School of Medicine.

\section{Measurements}

Baseline assessment of the OMCHS included a set of two selfadministered questionnaires. Also, a self-administered questionnaire was used in both the second and third survey. Participants mailed the answered questionnaires to the data management centre at the time of each survey. Research technicians completed missing or illogical data by telephone interview.

One of the self-administered questionnaires at baseline was a semi-quantitative, comprehensive, diet history questionnaire (DHQ) that assessed dietary habits during the preceding month $[15,16]$. Estimates of daily intake of foods (150 items in total), energy and selected nutrients were calculated using an ad hoc computer algorithm for the DHQ $[15,16]$, based on the Standard Tables of Food Composition in Japan [17, 18]. Total dairy product intake was considered as the sum of milk, yoghurt and cheese. Information on dietary supplements was not used because only a small number of participants used calcium (5.5\%) and multivitamin (4.2\%) supplements at least once per week. According to a validation study of 92 females aged 31-69 yrs, Pearson's correlation coefficients between the DHQ and 16-day weighed dietary records were 0.51 for calcium and 0.47 for vitamin D (unpublished data). Energyadjusted intake by the residual method was used for the analyses [19].

A second questionnaire at baseline asked about maternal age, gestation, family income, maternal and paternal education, maternal and paternal history of asthma, atopic eczema, and allergic rhinitis, and changes in diet in the previous 1 month. A paternal or maternal history of asthma, atopic eczema or allergic rhinitis was defined as positive if the respective parent had been treated with medications for any of these allergic disorders at some time prior to the start of the baseline survey.
A self-administered questionnaire in the second survey elicited information on the baby's sex, birth weight, date of birth, number of older siblings, maternal smoking during pregnancy and smoking in the household. A self-administered questionnaire in the third survey included questions on breastfeeding duration in months and symptoms of wheeze and eczema based on the International Study of Asthma and Allergies in Childhood (ISAAC) phase-I questionnaire [20, 21]. Wheeze was defined as present if the mother answered "yes" to the question "Has your child had wheezing or whistling in the chest in the last 12 months?". For eczema, affirmative answers to the following three questions were required: "Has your child ever had an itchy rash which was coming and going for at least 6 months?", "Has your child had this itchy rash at any time in the last 12 months?" and "Has this itchy rash at any time affected any of the following places: the folds of the elbow, behind the knees, in front of the ankles, under the buttocks, or around the neck, ears or eyes?".

\section{Statistical analysis}

Intake of dietary factors under study was categorised at quartile points based on the distribution in 763 subjects. Maternal age, gestation at baseline, residential municipality at baseline, family income, maternal and paternal education, maternal and paternal history of asthma, atopic eczema and allergic rhinitis, changes in maternal diet in the previous 1 month, season when data at baseline were collected, maternal smoking during pregnancy, older siblings, sex, birth weight, household smoking in the same room, breastfeeding duration and age at the third survey were $a$ priori selected as potential confounding factors.

Estimations of crude ORs and their 95\% CI of wheeze and eczema for each category of dietary intake under study in comparison with the lowest intake category were made by means of logistic regression analysis. Multiple logistic regression analysis was used to adjust for potential confounding factors. Trend of association was assessed by a logistic regression model assigning consecutive integers (1-4) to the quartiles of the exposure variables. All computations were performed using the SAS software package version 9.1 (SAS Institute, Inc., Cary, NC, USA).

\section{RESULTS}

In the third survey, the prevalence of wheeze and eczema based on the ISAAC criteria was $22.1 \%$ and $18.6 \%$, respectively, among 763 infants. About $75 \%$ of infants had been breastfed for 6 months or longer (table 1). Slight or substantial changes in diet in the previous 1 month were reported by 551 mothers due to nausea gravidarum $(n=453)$, maternal and fetal health $(n=89)$ and other reasons $(n=9)$. Maternal mean daily total energy consumption and energy-adjusted intake of total dairy products, calcium and vitamin D during pregnancy were $7628.6 \mathrm{~kJ}, 168.8 \mathrm{~g}, 542.3 \mathrm{mg}$, and $6.2 \mu \mathrm{g}$, respectively (table 2).

Compared with maternal total dairy product intake during pregnancy in the first quartile, consumption in the fourth quartile was significantly associated with a decreased risk of wheeze in the offspring, showing a clear inverse exposureresponse relationship: the adjusted OR for comparison of the highest with the lowest quartile was 0.45 (95\% CI: 0.25-0.79, p for trend 0.007) (table 3). Likewise, higher maternal intake of 


\begin{tabular}{|c|c|c|}
\hline TABLE 1 & \multicolumn{2}{|c|}{$\begin{array}{l}\text { Distribution of selected characteristics of } 763 \\
\text { parent-child pairs }\end{array}$} \\
\hline \multicolumn{3}{|c|}{ Baseline characteristics } \\
\hline \multicolumn{2}{|c|}{ Maternal age yrs } & $30.0 \pm 4.0$ \\
\hline \multicolumn{2}{|c|}{ Gestational age at baseline weeks } & $17.7 \pm 6.7$ \\
\hline \multicolumn{3}{|c|}{ Residential municipality } \\
\hline \multicolumn{2}{|c|}{ Neyagawa City } & 457 (59.9) \\
\hline \multicolumn{2}{|c|}{ Other than Neyagawa City } & $306(40.1)$ \\
\hline \multicolumn{3}{|c|}{ Family income $¥ \cdot \mathrm{yr}^{-1}$} \\
\hline \multicolumn{2}{|c|}{$<4,000,000$} & $211(27.7)$ \\
\hline \multicolumn{2}{|c|}{$4,000,000-5,999,999$} & $313(41.0)$ \\
\hline \multicolumn{2}{|c|}{$\geqslant 6,000,000$} & 239 (31.3) \\
\hline \multicolumn{3}{|c|}{ Maternal education yrs } \\
\hline \multicolumn{2}{|c|}{$<13$} & $212(27.8)$ \\
\hline \multicolumn{2}{|l|}{$13-14$} & $329(43.1)$ \\
\hline \multicolumn{2}{|c|}{$\geqslant 15$} & $222(29.1)$ \\
\hline \multicolumn{3}{|c|}{ Paternal education yrs } \\
\hline \multicolumn{2}{|c|}{$<13$} & $289(37.9)$ \\
\hline \multicolumn{2}{|l|}{$13-14$} & $127(16.6)$ \\
\hline \multicolumn{2}{|l|}{$\geqslant 15$} & $347(45.5)$ \\
\hline \multicolumn{2}{|c|}{ Maternal history of asthma } & $80(10.5)$ \\
\hline \multicolumn{2}{|c|}{ Maternal history of atopic eczema } & $120(15.7)$ \\
\hline \multicolumn{2}{|c|}{ Maternal history of allergic rhinitis } & $256(33.6)$ \\
\hline \multicolumn{2}{|c|}{ Paternal history of asthma } & $64(8.4)$ \\
\hline \multicolumn{2}{|c|}{ Paternal history of atopic eczema } & $72(9.4)$ \\
\hline Paternal his & ry of allergic rhinitis & $143(18.7)$ \\
\hline Changes in & liet in the previous 1 month & \\
\hline Never or & eldom & $212(27.8)$ \\
\hline Slight & & $336(44.0)$ \\
\hline Substant & & $215(28.2)$ \\
\hline Season wh & data were collected & \\
\hline Spring & & $241(31.6)$ \\
\hline Summer & & $127(16.6)$ \\
\hline Autumn & & $167(21.9)$ \\
\hline Winter & & 228 (29.9) \\
\hline Characterist & s at the post-natal assessmen & \\
\hline Maternal sn & king during pregnancy & 97 (12.7) \\
\hline One or mo & older siblings & 381 (49.9) \\
\hline Male sex & & $403(52.8)$ \\
\hline Birth weigh & & $3071.0 \pm 415.5$ \\
\hline Household & noking in same room as infant & $195(25.6)$ \\
\hline Breastfeedi & duration months & \\
\hline$<6$ & & $189(24.8)$ \\
\hline$\geqslant 6$ & & $574(75.2)$ \\
\hline Age of infa & at third survey months & \\
\hline $16-19$ & & $263(34.5)$ \\
\hline 20 & & $370(48.5)$ \\
\hline $21-24$ & & $130(17.0)$ \\
\hline
\end{tabular}

Data are presented as mean \pm SD or $n(\%)$.

milk and cheese during pregnancy was independently related to a reduced risk of wheeze in the children: the adjusted ORs for the highest versus lowest quartiles were 0.50 (95\% CI: 0.28-0.87, p for trend 0.02) and 0.51 (95\% CI: 0.31-0.85, p for trend 0.02$)$, respectively. With regard to different types of milk, maternal consumption of full-fat, but not low-fat, milk was significantly inversely related to the risk of infantile wheeze. No evident relationship was observed between maternal
TABLE 2 Distribution of daily intake of dairy products, calcium, and vitamin D in 763 pregnant females at baseline

$\begin{array}{lc}\text { Total energy kJ } & 7628.6 \pm 1924.1 \\ \text { Total dairy products } \mathbf{g} & 168.8 \pm 119.7 \\ \text { Milk g } & 122.6 \pm 108.8 \\ \text { Yoghurt g } & 40.5 \pm 39.8 \\ \text { Cheese g } & 5.7 \pm 8.5 \\ \text { Calcium } \mathbf{~ m g} & 542.3 \pm 170.7 \\ \text { Vitamin } \mathbf{D} \boldsymbol{\mu g} & 6.2 \pm 3.7\end{array}$

Data are presented as mean $\pm S D$. Nutrient and food intake were adjusted for total energy intake using the residual method.

yoghurt consumption during pregnancy and the risk of wheeze in the offspring. Maternal calcium intake during pregnancy was independently inversely associated with the risk of wheeze: the adjusted OR between extreme quartiles was 0.57 (95\% CI: 0.32-0.99, p for trend 0.04). Compared with maternal vitamin D intake during pregnancy in the first quartile, only its consumption in the second quartile was independently related to a decreased risk of wheeze in the children.

Maternal intake of total dairy products, milk, yoghurt, cheese and calcium during pregnancy was not measurably associated with the risk of eczema in the offspring. There was an inverted J-shaped association between maternal vitamin D intake during pregnancy and the risk of eczema in the children although adjusted ORs for the second and third quartiles were of borderline significance.

Because all adjusted ORs for infantile wheeze and eczema associated with maternal vitamin D intake during pregnancy in the second, third and fourth quartiles were below unity, maternal vitamin D consumption during pregnancy was categorised into two groups using a cut-off point at the 25th percentile $\left(<4.309\right.$ and $\left.\geqslant 4.309 \mu \mathrm{g} \cdot \mathrm{day}^{-1}\right)$. The consumption of $\geqslant 4.309 \mu \mathrm{g} \cdot$ day $^{-1}\left(\geqslant 172.4 \mathrm{IU} \cdot\right.$ day $\left.^{-1}\right)$ was significantly associated with a decreased risk of both wheeze and eczema in the offspring: the adjusted ORs were 0.64 (95\% CI 0.43-0.97) and 0.63 (95\% CI 0.41-0.98), respectively. No interaction was found in the association of maternal calcium intake during pregnancy with infantile wheeze between children whose mothers had consumed $4.309 \mu \mathrm{g}$ or more of vitamin $\mathrm{D}$ daily during pregnancy and those whose mothers had consumed $<4.309 \mu$ g daily $(\mathrm{p}=0.77$ for homogeneity of OR for the highest quartile).

Maternal intake of total dairy products, milk and cheese was correlated with maternal calcium consumption: Pearson's correlation coefficients were $0.85(\mathrm{p}<0.0001), 0.74(\mathrm{p}<0.0001)$ and $0.39(p<0.0001)$, respectively. The significant inverse relationships between maternal consumption of total dairy products, milk and cheese in the highest quartile and the risk of wheeze in the children were completely attenuated by further adjustment for maternal calcium intake during pregnancy as a continuous variable; the further-adjusted ORs were 0.68 (95\% CI 0.30-1.53), 0.74 (95\% CI 0.36-1.50) and 0.63 (95\% CI 0.37-1.07), respectively. 


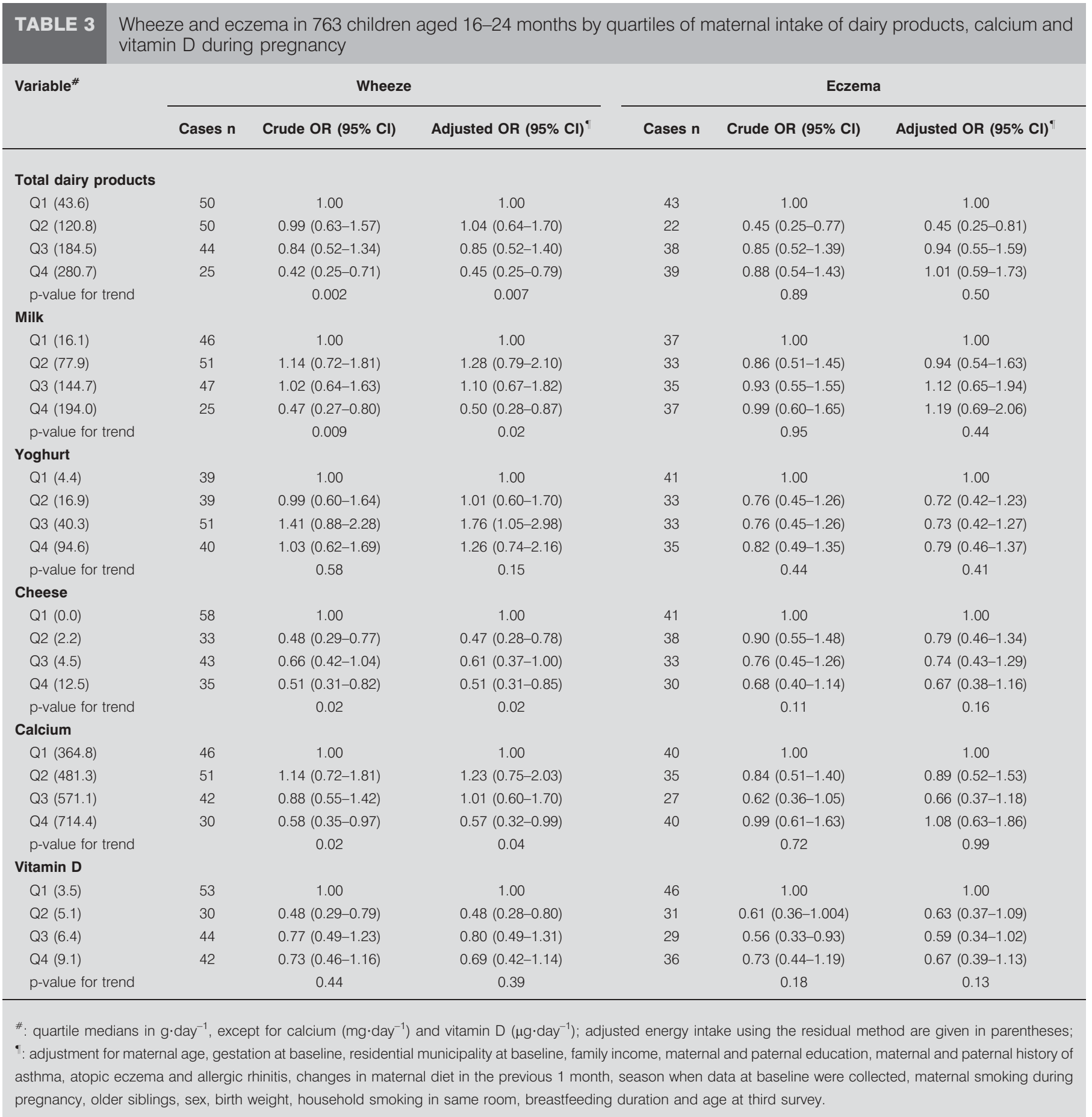

Maternal intake of calcium and vitamin D was correlated with maternal consumption of docosahexaenoic acid and vitamin E (table 4). Our previous findings using data from the OMCHS showed significant inverse associations between maternal intake of $\alpha$-linolenic acid, docosahexaenoic acid [22] and vitamin E [23] during pregnancy and the risk of wheeze in the infants aged 16-24 months. Additional adjustment for maternal intake of $\alpha$-linolenic acid, docosahexaenoic acid or vitamin E during pregnancy did not materially alter the significant inverse relationships between maternal intake of total dairy products, milk and cheese during pregnancy and the risk of wheeze in children. However, the inverse associations between maternal intake of calcium in the highest quartile and $\geqslant 4.309 \mu \mathrm{g} \cdot$ day $^{-1}$ of vitamin $\mathrm{D}$ and infantile wheeze were not statistically significant after further control for maternal intake of docosahexaenoic acid (further adjusted ORs (95\% CIs) were 0.60 (0.34-1.04) and 0.76 (0.47-1.25), respectively) or vitamin $\mathrm{E}$ (further adjusted ORs (95\% CIs) were 0.62 (0.35-1.08) and 0.71 (0.47-1.08), respectively) whereas the significant inverse associations remained after additional adjustment for maternal $\alpha$-linolenic acid intake. According to our previous findings regarding eczema, additional adjustment 
for maternal intake of n-6 polyunsaturated fatty acids [22], green and yellow vegetables, citrus fruit, or $\beta$-carotene [23] did not materially change the significant inverse association between maternal intake of $4.309 \mu \mathrm{g} \cdot \mathrm{day}^{-1}$ or more of vitamin D and infantile eczema whereas the inverse association had disappeared after further control for maternal vitamin $\mathrm{E}$ intake (further adjusted OR (95\% CI) was 0.68 (0.44-1.06)).

\section{DISCUSSION}

To the best of our knowledge, the current birth cohort study is the first to find that maternal intake of total dairy products, milk, especially full-fat milk, cheese and calcium during pregnancy was independently related to a decreased risk of wheeze in infants aged 16-24 months although such an inverse association with maternal yoghurt intake was not observed. On the other hand, no material associations were observed between maternal consumption of total dairy products, milk, yoghurt, cheese and calcium during pregnancy and the risk of eczema in the offspring. Children whose mother had consumed daily $4.309 \mu \mathrm{g}$ or more of vitamin D during pregnancy had a significantly reduced risk of wheeze and eczema, suggesting a threshold effect rather than a monotonic trend. This is the first study in a non-Western population to examine the relationship of maternal vitamin $\mathrm{D}$ intake to allergic disorders in the offspring.

The potential protective effects of maternal consumption of total dairy products, milk and cheese during pregnancy on wheeze in infants essentially disappeared when we additionally controlled for maternal calcium intake during pregnancy in this study. Thus, the beneficial associations with dairy foods might be attributable to some extent to calcium or unmeasured constituents associated with calcium. Unknown factors in yoghurt might have interfered with the expected benefit of maternal calcium intake during pregnancy in the development of wheeze in the offspring in our population. All calcium in bodily fluids, whether in blood, in interstitial spaces or within the cell cytosol or organelles, originates from the diet [24]. Several routes of $\mathrm{Ca}^{2+}$ influx may be implemented at various stages of the development and maturation of lymphocytes [25]. HAMMOND et al. [26] observed that increases in extracellular calcium caused $\mathrm{Ca}^{2+}$ to be released from intracellular stores in human B cells, activated signalling cascades and resulted in increased cluster of differentiation (CD)83 expression and responsiveness to Toll-like receptor, B-cell receptor and cytokine receptor signals. Local changes in extracellular calcium may be sensed by nearby B cells and prepare them to interact with $\mathrm{T}$ cells [26]. Thus, higher maternal calcium intake during pregnancy might be expected to moderate extracellular calcium fluctuations in a fetus and modulate antenatal immune responses via downregulation of the calcium-sensing mechanism on B cells, resulting in a decreased risk of allergic disorders in the child. However, no association was found between maternal calcium intake during pregnancy and the risk of eczema in infants in the current study.

The average vitamin D intake by pregnant females participating in a previously cited UK birth cohort study and in a US birth cohort study was $137 \mathrm{IU} \cdot$ day $^{-1}$ and $548 \mathrm{IU} \cdot$ day $^{-1}$, respectively $[11,12]$. The corresponding figure for the current study was $248 \mathrm{IU} \cdot$ day $^{-1}$. Milk fortified with vitamin D is the main source of vitamin D intake in the US diet [11]. Fish and eggs are major sources of vitamin D intake among Japanese people. An inverse relationship between maternal vitamin $\mathrm{D}$ intake during pregnancy and the risk of childhood wheeze was observed in the three different populations regardless of the amount of vitamin $\mathrm{D}$ intake. The main source of vitamin $\mathrm{D}$ is the cutaneous synthesis induced by sunlight exposure irrespective of populations [27]. The two previous birth cohort studies [11, 12] and our study did not take sunlight exposure status into consideration. Another UK prospective study reported that children whose mothers had higher serum concentrations of 25 $(\mathrm{OH})$-vitamin D in late pregnancy had a significantly increased risk of visible eczema on examination at 9 months and asthma at age 9 yrs [28]. The effects of vitamin D on allergic disorders might depend on the timing of vitamin D exposure. In a birth cohort study in Finland, subjects who had received vitamin D supplementation regularly during the first year compared to others had a marginally significantly increased risk of asthma at age 31 yrs [29]. Higher vitamin D intake at 5 months old was significantly positively related to the risk of eczema at the age of 6 yrs in Swedish children [30].

The present study had methodological strengths. Study subjects were homogeneous in terms of having the same residential background. We incorporated extensive information on potential confounding factors. The prospective design was likely to reduce the possibility of recall bias. The definition of wheeze and eczema was based on the ISAAC questions although validation tests of the ISAAC questions have not been

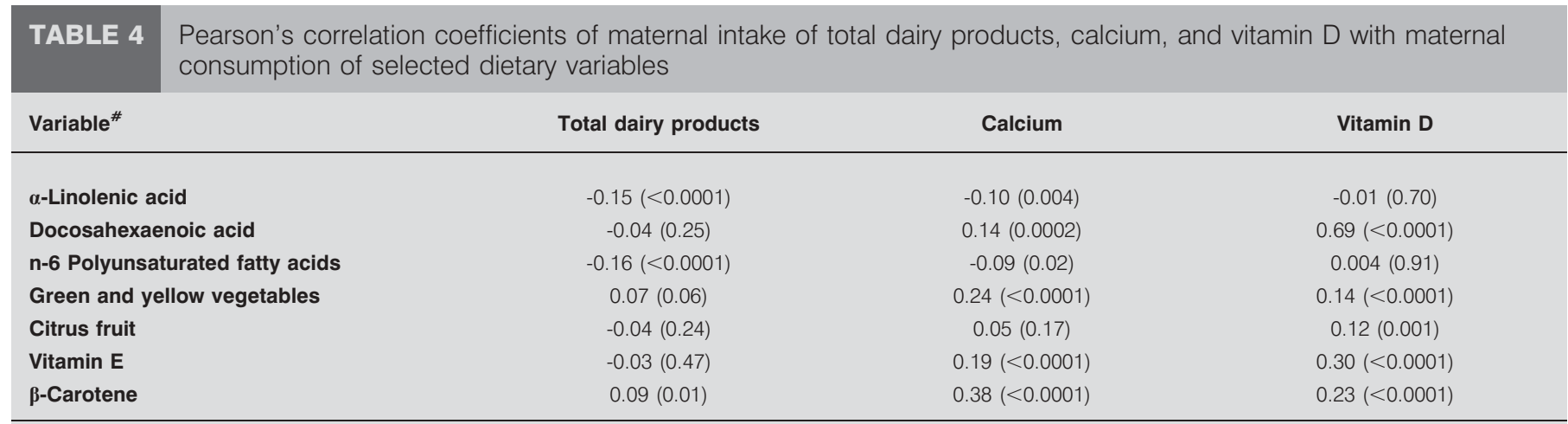

Data are presented as Pearson's correlation coefficient ( $\mathrm{p}$-value) ${ }^{\#}$ : nutrient and food intake were adjusted for total energy intake using the residual method. 
performed for Japanese infants. No attempt was made to ascertain outcome status through reviews of medical records. Moreover, the outcomes under study were measured at varying ages between 16 and 24 months and, additionally, wheeze in infancy is known not to be a good predictor of asthma over the longer term. These disadvantages could bias the magnitude of the observed effects towards the null.

There are other limitations in this study that deserve recognition. Our DHQ could only approximate consumption although the correlation between the DHQ and dietary records was reasonable, as described above. The consequence would have been an underestimation of values in our results. Our DHQ was designed to assess recent dietary intake, i.e. for 1 month prior to completing the questionnaire. This disadvantage is likely to be alleviated after adjustment for the season when data were collected, however. Changes in diet in the past 1 month were controlled for because pregnant females are likely to change their diet. Information on unpasteurised milk or raw milk was not included in our DHQ; however, Japanese people rarely consume raw milk even in rural areas. We did not include data on whether any of the participants lived on a farm, where consumption of raw milk might be more likely than in urban areas. At baseline, almost all of the participants lived in the Osaka Prefecture, an urban area that has the population density with 4,655 people per square kilometre; thus minimising the inclusion of farm families. The observed inverse associations with maternal intake of calcium and $\geqslant 4.309 \mu \mathrm{g} \cdot$ day $^{-1}$ vitamin D were attenuated by further adjustment for maternal consumption of docosahexaenoic acid or vitamin E. Thus, maternal consumption of docosahexaenoic acid or vitamin E might have confounded the inverse associations. Although we adjusted for a variety of potential confounders, we cannot eliminate the possibility that the observed inverse associations are a consequence of residual confounding by uncontrolled dietary or nondietary factors.

The follow-up rate was not good. Of the 1,002 participants at baseline, 239 mother-child pairs did not take part in the third survey. There were no material differences between the 239 nonparticipants and the 763 participants in the third survey with regard to distribution of maternal age, maternal and paternal history of asthma, atopic eczema and allergic rhinitis, and maternal intake of total energy, milk, yoghurt, cheese and calcium. Compared with non-participants in the third survey, participants were less likely to report low family income and a low maternal and paternal educational level and were more likely to have a high intake of vitamin D. Moreover, at baseline, the participation rate in Neyagawa City was only $17.2 \%$ and in other areas the participation rate could not be calculated because the exact number of eligible subjects among the sources from which participants were recruited was not available. Thus, the mother-child pairs in this study were likely not representative of Japanese mother-child pairs in the general population. In fact, educational levels were higher in the mothers in our study than in the general population. According to the year 2000 population census of Japan, the proportions of females aged 30-34 yrs in the Osaka Prefecture with $<13,13-14, \geqslant 15$ yrs and unknown number of years of education, were 49.2, 32.3, 13.6 and $4.9 \%$, respectively [31]. The present population might have had a greater awareness about health than the general population.
In summary, findings from this prospective study suggest that higher maternal consumption of total dairy products, milk, cheese, calcium and vitamin D during pregnancy may reduce the risk of infantile wheeze. Also, higher maternal vitamin D intake during pregnancy may be protective against eczema in the children. Our data, however, did not provide evidence that yoghurt intake during pregnancy is preventive against infantile wheeze and eczema. Because of a lack of inverse relationships between maternal intake of total dairy products, milk, cheese and calcium during pregnancy, and the risk of infantile eczema, we cannot rule out the possibility that such intake might be preventive against respiratory infections, rather than atopy. Further confirmation of these findings is required.

\section{SUPPORT STATEMENT}

The study was supported by a Grant-in-Aid $(13770206,16790351)$ for Scientific Research from the Ministry of Education, Culture, Sports, Science, and Technology and Health and Labour Sciences Research Grants, Research on Allergic Disease and Immunology from the Ministry of Health, Labour, and Welfare, Japan.

\section{STATEMENT OF INTEREST}

Statements of interest for Y. Miyake and K. Tanaka can be found at www.erj.ersjournals.com/misc/statements.dtl

\section{ACKNOWLEDGEMENTS}

The authors would like to acknowledge the Neyagawa City Government, Hirakata City Government, Katano City Government, Shijonawate City Government, Kaizuka City Government, Takaishi City Government, Hannan City Government, Neyagawa City Medical Association, Hirakata City Medical Association and the Kadoma City Medical Association (all Japan) for their valuable support.

\section{REFERENCES}

1 Kim JL, Elfman L, Mi Y, et al. Current asthma and respiratory symptoms among pupils in relation to dietary factors and allergens in the school environment. Indoor Air 2005; 15: 170-182.

2 Wijga AH, Smit HA, Kerkhof M, et al. Association of consumption of products containing milk fat with reduced asthma risk in preschool children: the PIAMA birth cohort study. Thorax 2003; 58 : 567-572.

3 Hijazi N, Abalkhail B, Seaton A. Diet and childhood asthma in a society in transition: a study in urban and rural Saudi Arabia. Thorax 2000; 55: 775-779.

4 Fussman C, Todem D, Forster J, et al. Cow's milk exposure and asthma in a newborn cohort: repeated ascertainment indicates reverse causation. J Asthma 2007; 44: 99-105.

5 Tabak C, Wijga AH, de Meer G, et al. Diet and asthma in Dutch school children (ISAAC-2). Thorax 2006; 61: 1048-1053.

6 Hong SJ, Lee MS, Lee SY, et al. High body mass index and dietary pattern are associated with childhood asthma. Pediatr Pulmonol 2006; 41: 1118-1124.

7 Farchi S, Forastiere F, Agabiti N, et al. Dietary factors associated with wheezing and allergic rhinitis in children. Eur Respir J 2003; 22: 772-780.

8 Woods RK, Walters EH, Raven JM, et al. Food and nutrient intakes and asthma risk in young adults. Am J Clin Nutr 2003; 78: 414-421.

9 Sausenthaler S, Koletzko S, Schaaf B, et al. Maternal diet during pregnancy in relation to eczema and allergic sensitization in the offspring at 2 y of age. Am J Clin Nutr 2007; 85: 530-537.

10 Willers SM, Wijga AH, Brunekreef B, et al. Maternal food consumption during pregnancy and the longitudinal development of childhood asthma. Am J Respir Crit Care Med 2008; 178: 124-131. 
11 Camargo CA Jr, Rifas-Shiman SL, Litonjua AA, et al. Maternal intake of vitamin D during pregnancy and risk of recurrent wheeze in children at 3 y of age. Am J Clin Nutr 2007; 85: 788-795.

12 Devereux G, Litonjua AA, Turner SW, et al. Maternal vitamin D intake during pregnancy and early childhood wheezing. Am J Clin Nutr 2007; 85: 853-859.

13 Litonjua AA, Weiss ST. Is vitamin D deficiency to blame for the asthma epidemic? J Allergy Clin Immunol 2007; 120: 1031-1035.

14 Miyake Y, Tanaka K, Sasaki S, et al. Breastfeeding and atopic eczema in Japanese infants: The Osaka Maternal and Child Health Study. Pediatr Allergy Immunol 2009; 20: 234-241.

15 Sasaki S, Yanagibori R, Amano K. Self-administered diet history questionnaire developed for health education: a relative validation of the test-version by comparison with 3-day diet record in females. J Epidemiol 1998; 8: 203-215.

16 Sasaki S, Ushio F, Amano K, et al. Serum biomarker-based validation of a self-administered diet history questionnaire for Japanese subjects. J Nutr Sci Vitaminol 2000; 46: 285-296.

17 Science and Technology Agency. Standard Tables of Food Composition in Japan. 5th revised and enlarged edn (in Japanese). Tokyo, Printing Bureau of the Ministry of Finance, 2005.

18 Science and Technology Agency. Standard Tables of Food Composition in Japan, fatty acids section. 5th revised and enlarged edn (in Japanese). Tokyo, Printing Bureau of the Ministry of Finance, 2005.

19 Willett W, Stampfer MJ. Total energy intake: implications for epidemiologic analyses. Am J Epidemiol 1986; 124: 17-27.

20 The International Study of Asthma and Allergies in Childhood (ISAAC) Steering Committee. Worldwide variations in the prevalence of asthma symptoms: the International Study of Asthma and Allergies in Childhood (ISAAC). Eur Respir J 1998; 12: 315-335.

21 Williams H, Robertson C, Stewart A, et al. Worldwide variations in the prevalence of symptoms of atopic eczema in the international study of asthma and allergies in childhood. J Allergy Clin Immunol 1999; 103: 125-138.

22 Miyake Y, Sasaki S, Tanaka K, et al. Maternal fat consumption during pregnancy and risk of wheeze and eczema in Japanese infants aged 16-24 months: The Osaka Maternal and Child Health Study. Thorax 2009; 64: 815-821.

23 Miyake Y, Sasaki S, Tanaka K, et al. Consumption of vegetables, fruit, and antioxidants during pregnancy and wheeze and eczema in infants. Allergy 2010; [Epub ahead of print DOI: 10.1111/j.13989995.2009.02267.x].

24 Lamprecht SA, Lipkin M. Chemoprevention of colon cancer by calcium, vitamin D and folate: molecular mechanisms. Nat Rev Cancer 2003; 3: 601-614.

25 Vig M, Kinet JP. Calcium signaling in immune cells. Nat Immunol 2009; 10: 21-27.

26 Hammond CM, White D, Tomic J, et al. Extracellular calcium sensing promotes human B-cell activation and function. Blood 2007; 110: 3985-3995.

27 Holick MF. Sunlight and vitamin D for bone health and prevention of autoimmune diseases, cancers, and cardiovascular disease. Am J Clin Nutr 2004; 80: Suppl. 6, 1678S-1688S.

28 Gale CR, Robinson SM, Harvey NC, et al. Maternal vitamin D status during pregnancy and child outcomes. Eur J Clin Nutr 2008; 62: 68-77.

29 Hyppönen E, Sovio U, Wjst $\mathrm{M}$, et al. Infant vitamin D supplementation and allergic conditions in adulthood: northern Finland birth cohort 1966. Ann N Y Acad Sci 2004; 1037: 84-95.

30 Bäck O, Blomquist HK, Hernell O, et al. Does vitamin D intake during infancy promote the development of atopic allergy? Acta Derm Venereol 2009; 89: 28-32.

31 Statistic Bureau, Ministry of Public Management, Home Affairs, Posts and Telecommunications. 2000 population census of Japan, Vol. 3-2-27, labour force status of population, industry (major groups) of employed persons, and education (in Japanese). Osakafu. Tokyo. Statistic Bureau, Ministry of Public Management, Home Affairs, Posts and Telecommunications, 2002. 